\title{
Graph Theory Towards Module-K Odd-Elegant Labelling Of Graphical Passwords
}

\author{
Xiaohui Zhang ${ }^{1}$, Hui Sun ${ }^{1}$, and Bing Yao ${ }^{1,2, \star}$ \\ ${ }^{1}$ College of Mathematics and Statistics, Northwest Normal University, Lanzhou, 730070 CHINA \\ ${ }^{2}$ School of Electronic and Information Engineering, Lanzhou Jiaotong University, Lanzhou 730070, CHINA
}

\begin{abstract}
Graph labellings have been applied in many areas of science and engineering, such as in the development of redundant arrays of independent disks which incorporate redundancy utilizing erasure codes, algorithms, design of highly accurate optical gauging systems for use on automatic drilling machines, design of angular synchronization codes, design of optimal component layouts for certain circuit-board geometries, and determining configurations of simple resistor networks which can be used to supply any of a specified set of resistance values. Based on the idea of of "topological structure plus number theory" and magic type of various labellings for solving network transfer delay by using other type of graphical passwords. We define a new graph labelling, called Module-K odd-elegant labelling, and find some network models that admit our new labelling, and furthermore our methods can be transformed into effective algorithms.
\end{abstract}

\section{Introduction}

No report tells us that much of graphical passwords were applied to business and practice (Ref. [1], [2], [3]). An idea of "topological structure plus number theory" (Topsnut) as an alternative the existing graphical passwords is proposed in [6], which can be realized by graph labellings, and this idea is related with many mathematical conjectures, such as "Every tree is graceful" due to Rosa [4]. The survey article [5] has collected over 1700 papers on research of graph labellings. Motivated from the some graph labellings we define a new labeling, called Module-K odd-elegant labelling. The Module-K odd-elegant labelling can induces some mathematical problems, such as: Every tree admits a Module-K odd-elegant labelling. Furthermore, we can plant this new labelling to other graph labellings, see [7], strongly graceful labelling in [10], odd-graceful labelling in [11], oddelegant labelling in [18], seven other labellings in [13] and so on, which mean that exploring new graphical passwords will bring more new mathematical subjects and new problems. An idea of "topological structure plus number theory" for creating new type of graphical passwords was proposed firstly by Wang et al. in [14] and [15]. In comparing with those graphical passwords mentioned in [16], the new graphical passwords obtained by Topsnut needs less storage and implements quickly in network communication.

\section{Preliminary and new labellings}

We use standard notation and terminology of graph theory. Graphs mentioned are loopless, no multiple edges, undirected, connected and finite, unless otherwise specified. A $(p, q)$-graph

^e-mail: yybb918@163.com 
$G$ is one with $p$ vertices and $q$ edges. The shorthand symbol $[m, n]$ stands for an integer set $\{m, m+1, \ldots, n\}$, where $m$ and $n$ are integers with $0 \leq m<n$; the notation $[s, t]^{o}$ indicates an oddset $\{s, s+2, \ldots, t\}$, where $s$ and $t$ both are odd integers with $1 \leq s<t$; and the notation $[k, \ell]^{e}$ indicates an even-set $\{k, k+2, \ldots, \ell\}$, where $k$ and $\ell$ both are even integers with $0 \leq k<\ell$. Graphical password will be abbreviated as GPW, and "Topsnut-GPW" is the abbreviation of "graphical passwords based on the idea of topological structure plus number theory".

Definition 1 [5] If a $(p, q)$-graph $G$ admits a mapping $f: V(T) \rightarrow[0, q]$ such that $f(u) \neq f(v)$ for distinct $u, v \in V(G)$, and the edge labelling set $\{f(u v)=|f(u)-f(v)|: u v \in E(G)\}=[1, q]$, then $f$ is called a graceful labelling, and $G$ is called a graceful graph.

We write the vertex labelling set $f(V(G))=$ $\{f(u): u \in V(G)\}$ and the edge labelling set $f(E(G))=\{f(u v): u v \in E(G)\}$ hereafter. Suppose that a bipartite graph $G$ admits a graceful labelling $f$ such that $\max \{f(x): x \in X\}<$ $\min \{f(y): y \in Y\}$, where $(X, Y)$ is the bipartition of $V(G)$, we call $f$ a set-ordered graceful labelling, and this case is denoted as $f(X)<f(Y)$ for short $([17,18])$.

Definition 2 [9] Suppose that a (p,q)-graph $G$ admits a mapping $f: V(G) \rightarrow[0,2 q-1]$ such that $f(u) \neq f(v)$ for distinct $u, v \in V(G)$, and the label $f(u v)$ of every edge $u v \in E(G)$ is defined as $f(u v)=f(u)+f(v)(\bmod 2 q)$ and the set of all edge labels is equal to $[1,2 q-1]^{\circ}$, we call $f$ an odd-elegant labelling and $G$ to be odd-elegant.

Definition 3 [9] Let $\left(V_{1}, V_{2}\right)$ be the bipartition of a bipartite $(p, q)$-graph $G$. If $G$ admits an oddelegant labelling $f$ such that $\max \{f(u): u \in$ $\left.V_{1}\right\}<\min \left\{f(v): \quad v \in V_{2}\right\}$, then we call $f a$ set-ordered odd-elegant labelling, and write this case as $f\left(V_{1}\right)<f\left(V_{2}\right)$.

Motivated from the above graph labellings we define a new Module-K odd-elegant labelling as follows.
Definition 4 Suppose that a $(p, q)$-graph $G$ admits a mapping $f: V(G) \rightarrow[0,2 q-1]$ such that $f(u) \neq f(v)$ for distinct $u, v \in V(G)$, and the label $f(u v)$ of every edge $u v \in E(G)$ is defined as $f(u v)=f(u)+f(v)+K(\bmod 2 q)$ for some given $K$, and the set of all edge labels is equal to $[1,2 q-1]^{o}$ or $[2,2 q]^{e}$, we call $f a$ Module-K odd-elegant labelling and $G$ to be Module-K odd-elegant. Moreover, if $\left(V_{1}, V_{2}\right)$ is the bipartition of $G$, and the Module-K oddelegant labelling $f$ obeys $\max \left\{f(u): u \in V_{1}\right\}<$ $\min \left\{f(v): v \in V_{2}\right\}$, then we call $f$ a set-ordered Module-K odd-elegant labelling, and write this case as $f\left(V_{1}\right)<f\left(V_{2}\right)$.

In the current paper, we make the following contributions to the literature on password security:

(1) be used conveniently in usually;

(2) with strong security, that is, it is difficult to be broken;

(3) there are enough graphs and labellings for making desired keys and locks.

For answering the above problems, we prove the series cryptographical graphs have good properties, and show the guarantee for constructing large scale of cryptographical trees from smaller cryptographical trees. The methods used for constructing the desired cryptographical graphs can be transformed into efficient algorithms.

\section{Main results and their proofs}

Theorem 1 A connected bipartite graph $G$ is set-ordered graceful if and only if there exists a $K$ such that $G$ admits a Module-Kodd-elegant labelling.

Proof. Necessity. Suppose that a bipartite graph $G$ admits a graceful labelling $f$ such that $\max \{f(x): x \in X\}<\min \{f(y): y \in Y\}$, where $(X, Y)$, we define $X=\left\{x_{i}: i \in[1, s]\right\}$ and $Y=\left\{y_{j}: j \in[1, t]\right\}$ with $s+t=p$ and $x_{i}, y_{j} \in V(G)$. Let $G$ have a set-ordered graceful labelling $f$ with $f\left(x_{i}\right)=i-1$ for $i \in[1, s]$ and $f\left(y_{j}\right)=s-1+j$ for $j \in[1, t]$, and $f\left(x_{i} y_{j}\right)=$ $f\left(x_{i}\right)-f\left(y_{j}\right)=s+j-i-2$ for each edge 
$x_{i} y_{j} \in E(T)$. Let $G$ have a labelling $g$ define as: $g\left(x_{i}\right)=2 f\left(x_{i}\right)$ for $i \in[1, s], g\left(y_{j}\right)=2 p-1-$ $2 f\left(y_{j}\right)$ for $i \in[1, t]$. for each edge $x_{i} y_{j} \in E(G)$, For some given $K \geq 0, g\left(x_{i}\right)+g\left(y_{j}\right)+K=$ $2 f\left(x_{i}\right)+2 p-1-2 f\left(y_{j}\right)+K=2(i+p+j-s)+K-1$, and $g\left(x_{i} y_{j}\right)=g\left(x_{i}\right)+g\left(y_{j}\right)+K(\bmod 2 q)$, which implies $g\left(x_{i} y_{j}\right) \in[1,2 p-1]^{o}$ or $[2,2 q]^{e}$, such that $g$ is Module-K odd-elegant labelling with for each edge $x_{i} y_{j} \in E(G)$.

Sufficiency. Suppose that $G$ admits a Module-K odd-elegant labelling $h$ such that $h\left(x_{i}\right)=2(i-1), i \in[1, s] ; h\left(y_{j}\right)=2 p-1-$ $2(s-1+j), j \in[1, t] . h\left(x_{i} y_{j}\right)=2(i+p-s-$ $j)-1+K(\bmod 2 q)$ for $i \in[1, s]$ and $j \in[1, t]$, and $h\left(x_{i} y_{j}\right) \in[1,2 q-1]^{o}$ or $[2,2 q]^{e}$. We define a labelling $f_{1}$ of $G$ as: $f_{1}\left(x_{i}\right)=\frac{1}{2} h\left(x_{i}\right)$ for $i \in[1, s] ; f_{1}\left(y_{j}\right)=h\left(y_{j}\right)-2 p+1$ for $j \in[1, t] ; f_{1}\left(x_{i} y_{j}\right)=h\left(x_{i} y_{j}\right)-K$ for $i \in[1, s]$ and $j \in[1, t]$. which produces $f_{1}(V(G))=[1, q]$ and $f_{1}\left(x_{i} y_{j}\right) \in[0, q]$. Thereby, $f_{1}$ is a set-ordered graceful labelling of $G$.
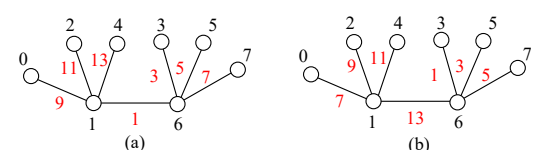

Figure 1. (a) A Module-8 odd-elegant labelling; (b) a Module-6 odd-elegant labelling.

A union graphical quasi-group $\bigcup_{i}(G)$ of order $n$ is defined as: $\bigcup_{i}(G)=\{G: V(G)=$ $1,2, \ldots, n\}$, the operation between elements of $\bigcup_{i}(G)$ is the graph union operation " $\bigcup$ ", and $G \cup H$ produces a simple graph of order $n$.

(i) (Zero element) the zero element of $\bigcup_{i}(G)$ is the unique complete graph $K_{n}$, since $G \cup K_{n}=K_{n}$.

(ii) (Inverse element) the complementary $G$ of each element $G \in \bigcup_{i}(G)$ is the inverse element of $G$, since $G \cup G=K_{n}$.

(iii) Uniqueness and closure. $G \cup H$ is unique and $G \cup H \in \bigcup_{i}(G)$ for any element $H \in \bigcup_{i}(G)$.

(iv) (Associated law) $\left(G_{1} \cup G_{2}\right) \cup G_{3}=$ $G_{1} \cup\left(G_{2} \cup G_{3}\right)$.

A graphical group obtained from the graph Module-K odd-elegant labelling. Let $f_{1}$ :
$V\left(G_{k}\right) \rightarrow[0,2 q-1]$ be a Module-K odd-elegant labelling such that the set of all edge labels is equal to $[1,2 q-1]^{o}$ or $[2,2 q]^{e}$ for a $(p, q)$-graph $G_{k}$. Define every edge $u v \in E\left(G_{k}\right)$ is defined as $f(u v)=f(u)+f(v)+K(\bmod 2 q)$ with some given $K$. Notice that $T_{i}(i \in[1, n])$ has identical vertex labelling of $G_{k}$. Each copy of the $(p, q)$ graph $G_{k}$ is written as $T_{i}(i \in[1, n])$. Define a 3element operation $T_{i} \oplus T_{j} \ominus T_{k}=T_{i+j-k}(\bmod K)$ We call this particular operation as the ModuleK odd-elegant additive-sum of graphs.
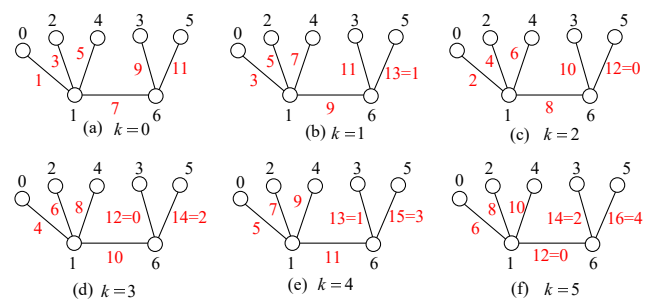

Figure 2. $T_{i}(i \in[1,4])$.

Corollary 1 The set Oele $(G)$ based on a $(p, q)$ graph $G_{1}$ forms an Abelian additive group, we call Oele $(G)$ a labelling graphical group based on the graph $G$ hereafter. Furthermore, $f(u v)=$ $f(u)+f(v)+K(\bmod 2 q)$ with some given $K$.

Proof. First of all, the equation $F\left(T_{i}\right) \oplus$ $F\left(T_{j}\right) \ominus F\left(T_{k}\right)=F\left(T_{i+j-k}(\bmod 2 q)\right.$ with $f(u v)(\bmod 2 q) \in[1,2 q-1]^{o}$ or $[2,2 q]^{e} f(u v)=$ $f(u)+f(v)+K(\bmod 2 q)$ follows the equation $\left[f_{i}(x)+f_{j}(x)+K\right](\bmod 2 q)$ with $V(G)=$ $V\left(G_{i}\right)=V\left(G_{j}\right)=V\left(G_{k}\right)$. We are ready to show four principles for a standard group in the following.

(1) Zero element. The zero element of $\operatorname{Oele}(G)$ is $F\left(T_{1}\right)$ as $k=0$ in the equation.

(2) Inverse element. The inverse of each element $T_{i} \in \operatorname{Oele}(G)$. In fact, $F\left(T_{i}\right) \oplus F\left(T_{j}\right) \ominus$ $F\left(T_{k}\right)=F\left(T_{i+j-k}(\bmod 2 q)\right)$.

(3) Uniqueness and closure. $F\left(T_{i}\right) \oplus F\left(T_{j}\right) \in$ $\operatorname{Oele}(G)$.

(4) Associative law. Since $\left[F\left(T_{i}\right) \oplus F\left(T_{j}\right)\right] \oplus$ $F\left(T_{l}\right)=F\left(T_{i+j-l}(\bmod 2 q)\right)$, so the associative law holds true.

We conclude that $\operatorname{Oele}(G)$ is a Module-K odd-elegant graphical group. Next, we determine a connection between $f_{1}(u v)=f_{1}(u)+$ 
$f_{1}(v)+K(\bmod 2 q)$ and $f_{k}(u)+f_{k}(v)+K(\bmod$ $2 q$ ) with some given $K$ in the following. Notice that $f_{1}\left(E\left(T_{1}\right)\right)=[1,2 q-1]^{o}$ or $[2,2 q]^{e}$. Consider the labelled graph $T_{k}$, take an edge $u v \in E\left(T_{k}\right)$, we have $f_{k}(u v)=f_{k}(u)+f_{k}(v)+K(\bmod 2 q)=$ $\left[f_{1}(u)+f_{1}(v)+(k-1)\right](\bmod 2 q)$, and there are the following cases: $f_{k}(u)+f_{k}(v)=f_{1}(u)+f_{1}(v)$. The proof of this theorem is complete.

Theorem 2 Let $G_{1}, G_{2}, \ldots, G_{m}$ be $m$ bipartite graphs having Module-K odd-elegant labellings and $\left(X_{i}, Y_{i}\right)$ be the bipartition of each $V\left(G_{i}\right)$ with $i \in[1, m]$. The graph $G$ is obtained by joining $a$ vertex of $G_{i}$ with a vertex of $G_{i+1}$ for $i \in[1, m]$. When $m$ is odd, Then $G$ has a Module-K oddelegant labelling.

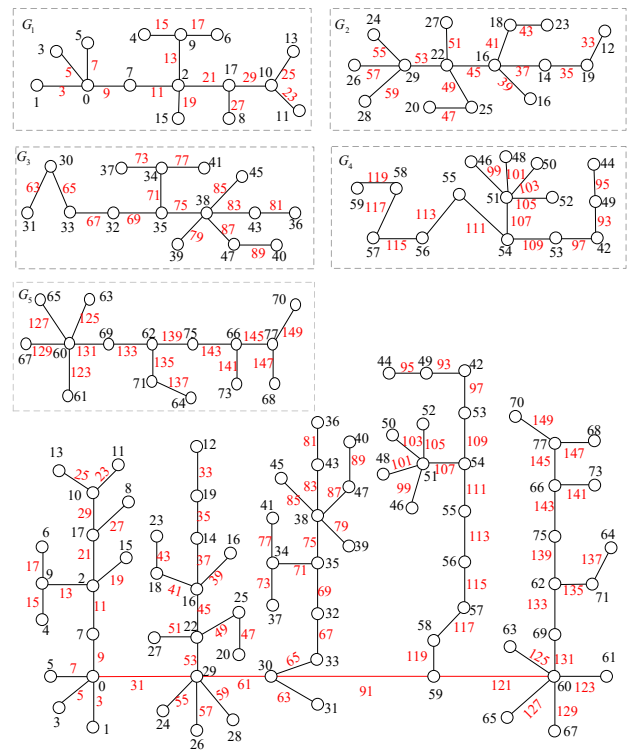

Figure 3. A Module-2 odd-elegant labelling for illustrating Theorem 2.

A dual labelling $f_{k}$ of a modular labelling $f_{k}$ is defined by $f_{k}(u v) \in[1,2 q-1]^{o}$ or $[2,2 q]^{e}$. Let $\operatorname{Oele}(G)$ be the set of the graphs $H_{k}$, where each $H_{k}$ is a labelled $\operatorname{Oele}(G)$ by the dual labelling $f_{k}$ of the modular labelling $f_{k}$ with $x \in V(G)$ with some given $K$. We propose two new graphical subject to some con-straint sets as: When $G_{1}, G_{2}, \ldots, G_{m}$ be $m$ bipartite graphs and neither $m$ is odd or even with the Module-K oddelegant labelling $g$ induced by the vertex setlabelling, we show that every simple, connected $(p, q)$-graph $G$ admits a Module-K odd-elegant labelling, and any tree having a Module-K oddelegant labelling. We propose the following problems for further researching new graphical passwords:

Theorem 3 Let $G_{1}, G_{2}, \ldots, G_{m}$ be $m$ bipartite graphs having Module-K odd-elegant labellings and $\left(X_{i}, Y_{i}\right)$ be the bipartition of each $V\left(G_{i}\right)$ with $i \in[1, m]$. The graph $G$ is obtained by joining $a$ vertex of $G_{i}$ with a vertex of $G_{i+1}$ for $i \in[1, m]$. Then $G$ has a Module-K odd-elegant labelling.

Theorem 4 Let $G_{1}, G_{2}, \ldots, G_{m}$ be $m$ bipartite graphs having Module-K odd-elegant labellings and $\left(X_{i}, Y i\right)$ be the bipartition of each $V\left(G_{i}\right)$ with $i \in[1, m]$. The graph $G$ is obtained by joining $a$ vertex of $G_{i}$ with a vertex of $G_{i+1}$ for $i \in[1, m]$. If $m$ is even, then $G$ has a Module-Kodd-elegant labelling.

\section{Conclusion and further researches}

We are working on new type of graphical passwords based on the idea of "topological structure plus number theory", vividly speaking, new graphical passwords can be called "mathematical fingerprints". And we are exploring transforming human faces into mathematical models in order to recognizing and comparing human faces through mathematical models rather than image recognition among vast of human faces, such works can be considered as "mathematical faces". However, we will make effort to let mathematical fingerprints approximating to human fingerprints. By our experience on working Topsnut-GPWs, we propose the following conjectures:

(i) Every bipartite graph admits a Module-K odd-elegant labelling.

(ii) A graph is odd-graceful if and only if it admits a Module-K odd-elegant labelling.

(iii) Any tree T has a Module-Kodd-elegant labelling. 


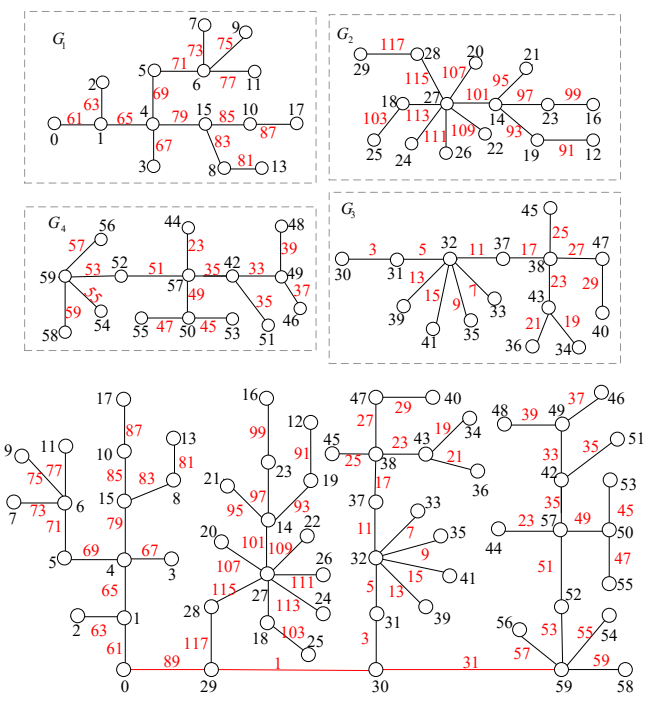

Figure 4. A Module-60 odd-elegant labelling for illustrating Theorem 4

Some of recent works have demonstrated that textual passwords are particularly vulnerable to targeted online guessing. Textual passwords may be influenced by personal information and textual passwords may be reused, both of which contribute to the vulnerability of textual passwords to online guessing. "Topological structure plus number theory" can be realized by graph labellings, future tests could be designed to answer this question.

\section{Acknowledgment}

This research was supported by the National Natural Science Foundation of China under grants No. 61163054, No. 61662066 and No. 61363060 .

\section{References}

[1] Xiaoyuan Suo, Ying Zhu, G. Scott. Owen. Graphical Password: A Survey. In: Proceedings of Annual Computer Security Applications Conference (ACSAC), Tucson, Arizona. IEEE (2005) 463-472.
[2] R. Biddle, S. Chiasson, and P.C. van Oorschot. Graphical passwords: Learning from the First Twelve Years. ACM Computing Surveys, 44(4), Article 19:1-41. Technical Report TR-09-09, School of Computer Science, Carleton University, Ottawa, Canada. 2009.

[3] Haichang Gao, Wei Jia, Fei Ye and Licheng Ma. A Survey on the Use of Graphical Passwords in Security. Journal Of Software, Vol. 8, No. 7, July 2013, 1678-1698.

[4] Rosa A. On certain valuations of the vertices of a graph. Theory of Graphs, 1967:349-355.

[5] Joseph A. Gallian. A Dynamic Survey of Graph Labeling. The electronic journal of combinatorics, 17 (2014), \# DS6.

[6] Hongyu Wang, Jin Xu, Bing Yao. Exploring New Cryptographical Construction Of Complex Network Data. IEEE First International Conference on Data Science in Cyberspace. IEEE Computer Society, (2016):155-160.

[7] Hongyu Wang, Bing Yao, Chao Yang, Sihua Yang, Xiang-en Chen, MingYao, Zhenxue Zhao. Edge-Magic Total Labellings Of Some Network Models. (Proceeding of ISCCCA13, Published by Atlantis Press, Paris,France, 2013, 0491-0494.) Applied Mechanics and Materials Vols. 347-350 (2013) pp 27522757.

[8] Hongyu Wang, Bing YAO, Chao YANG, Sihua YANG, Xiang'en CHEN. Labelling Properties Of Models Related with Complex Networks Based On Constructible Structures. Advanced Materials Research Vols. 765-767 (2013) pp 1118-1123. DOI:10.4028/www.scientific.net/AMR.765767.1118

[9] Hongyu Wang, Bing Yao, Ming Yao. Generalized Edge-Magic Total Labellings Of Models from researching Networks. Information Sciences 279 (2014) 460-467. DOI:10.1016/j.ins.2014.03.132

[10] Bing Yao, Hui Cheng, Ming Yao and Meimei Zhao, A Note on Strongly Graceful Trees. Ars Combinatoria 92 (2009), 155-169.

[11] Xiangqian Zhou, Bing Yao, Xiang-en Chen and Haixia Tao. A proof to the odd- 
gracefulness of all lobsters. Ars Combinatoria 103 (2012), 13-18.

[12] Xiangqian Zhou, Bing Yao, Xiang'en Chen. Every Lobster Is Odd-elegant. nInformation Processing Letters, 113 (2013), 3033.

[13] B. YAO, X. LIU and M. YAO. Connections Between Labellings Of Trees. Bulletin of the Iranian Mathematical Society, Vol. 43, No.2 (2017): 275-283.

[14] Hongyu Wang, Jin Xu,Bing Yao. The Key-models And Their Lock-models For Designing New Labellings Of Networks.Proceedings of 2016 IEEE Advanced Information Management, Communicates,Electronic and Automation Control Conference (IMCEC 2016): 565-5568.
[15] Hongyu Wang, Jin Xu,Bing Yao. Exploring New Cryptographical Con-struction Of Complex Network Data. IEEE First International Conference on Data Science in Cyberspace. IEEE Computer Society, (2016):155-160.

[16] Xiaoyuan Suo, Ying Zhu, G. Scott. Owen, Graphical Passwords: A Survey. In Annual Computer Security Applications Conference (ACSAC), December 2005: 463-472. DOI: 10.1109/CSAC.2005.27 Source: DBLP

[17] Bing Yao, Hui Cheng, Ming Yao, Meimei Zhao, A note on strongly graceful trees,Ars Combinator. 92 (2009) 155-169.

[18] Xiangqian Zhou, Bing Yao, Xiang'en Chen, Haixia Tao, A proof to the oddgracefulness of all lobsters, Ars Combinator. 103 (2012) 13-18. 